\title{
Determinantes de la pertenencia a entidades deportivas
}

\author{
WALESKA SIGÜENZA \\ Departamento Economía Financiera I, UNIVERSIDAD DEL PAÍS VASCO, ESPAÑA. E-mail: \\ waleska.siguenza@ehu.es
}

\section{PETR MARIEL}

Departamento Economía Aplicada III, UNIVERSIDAD DEL PAIS VASCO, ESPAÑA. E-mail: petr.mariel@ehu.es

\begin{abstract}
RESUMEN
La justificación del estudio reside en la importancia del deporte en nuestra sociedad y en el papel que juegan las entidades deportivas como catalizadores entre el deporte y las relaciones sociales. El objetivo del trabajo es analizar los factores socio-demográficos y socio-económicos que determinan el hecho de pertenecer a una entidad deportiva. La metodología de un modelo heteroscedástico de elección discreta binaria, junto con los datos obtenidos de la encuesta "Hábitos Deportivos de los Españoles" en 2005, permite analizar los efectos de distintas variables sobre la probabilidad de pertenecer a una entidad deportiva. Los resultados obtenidos muestran que en la demanda de pertenencia a una entidad deportiva influyen positivamente el género, el nivel estudios, la posesión de una buena forma física y la práctica de deporte, entre otros.
\end{abstract}

Palabras clave: Determinantes sociales, entidad deportiva, logit heteroscedástico.

\section{Factors Affecting Sport Organizations Membership}

\begin{abstract}
The main motivation for this study lies in the importance sports have in our nowadays society and, more specifically, in the role sport organizations play as merging factor between sports and social relations. The main objective of this study is the analysis of the variables that have an effect on the decision of an individual to become a member of a given sport club. The proposed methodology of using a heteroskedastic binary choice model, and its application to a data set obtained from the survey of Spanish sport habits collected in the year 2005, allows us to be able to obtain a detailed analysis of the effect the different variables set have on the probability to become a member of a sport club. Results show that the demand of a sport club membership is closely related to individual characteristics such as gender, level of education, physical fitness and if the individual is an athlete or not.
\end{abstract}

Keywords: Social Status Factors, Sport Club, Heteroskedastic Logit Model.

Clasificación JEL: C25, L83

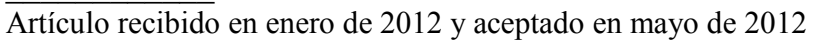

Artículo disponible en versión electrónica en la página www.revista-eea.net, ref. ə-30207 


\section{INTRODUCCIÓN}

En los últimos años se ha producido una evolución ascendente de la población que realiza actividades deportivas y del número de organizaciones que promueven y acogen su práctica. Podría incluso considerarse que, junto con las telecomunicaciones, el deporte constituye el fenómeno social más relevante de la segunda mitad del siglo XX. La actividad deportiva se ha convertido en un referente en las relaciones sociales y en un icono de aceptación social. Al mismo tiempo, esta práctica deportiva está condicionada por el grupo social y las condiciones socio-estructurales de la persona que la realiza (Erben et al., 1992). Actualmente los individuos no sólo practican deporte con un objetivo competitivo y de rendimiento físico, sino que, cada vez con mayor intensidad, se impone el denominado modelo "relacional" que, según Buñuel (1994), "está dominado por la relevancia de aspectos lúdicos y de relación social, así como por el deseo de construir espacios de comunicación opuestos a una lógica instrumentalista". También se puede observar un modelo "expresivo" (Puig y Heinemann, 1991) que consiste en prácticas deportivas poco organizadas y sometidas a procesos constantes de innovación y diversificación, como pueden ser los deportes californianos y los de aventura en la naturaleza. Y, por último, se pueden constatar prácticas deportivas destinadas al culto del cuerpo que se acercan al modelo instrumental (Buñuel, 1994).

De este modo, en la actualidad el deporte comienza a superar el ámbito reservado para aquellos que se dedican profesionalmente a su práctica y empieza a constituirse dentro de una concepción más amplia en la que se pueden incluir todo tipo de actividades. En la Carta Europea del Deporte, aprobada en Rodas (Grecia) en el año 1992, se establece que se entenderá por deporte "todo tipo de actividades físicas que, mediante una participación, organizada o de otro tipo, tengan por finalidad la expresión o la mejora de la condición física y psíquica, el desarrollo de las relaciones sociales o el logro de resultados en competiciones de todos los niveles". Además, la Carta Europea del Deporte fue revisada en 2001 pero la definición de deporte expuesta no se modificó.

Este cambio en la concepción de la actividad deportiva ha propiciado una importante reestructuración en las entidades deportivas que ya no se ajustan exclusivamente al perfil del deporte federado y del club tradicional. Dentro del concepto de organización deportiva se enmarcan una gran diversidad de organizaciones que trabajan en el sector del deporte (Slack y Parent, 2006): fabricantes de material deportivo, organizadores de eventos deportivos, empresas de comunicación relacionadas directamente con el deporte y las que promueven actividades deportivas, entre otras.

Este estudio se centra en aquellas organizaciones deportivas cuyo objetivo es la promoción y desarrollo del deporte (instalaciones municipales, clubes deportivos privados o públicos, gimnasios, etc.), dado el marcado carácter socio-de- 
portivo que poseen. Gómez et al. (2007) establecen que la misión de estas entidades deportivas es: "Satisfacer el interés de una comunidad por realizar actividad física y sociabilizar a través de la participación en actividades deportivas" e incluyen dentro del objetivo principal la labor de integración social a través del deporte. Asimismo, otros trabajos destacan el importante papel socializador que pueden jugar las entidades deportivas en la infancia y la adolescencia (Patriksson, 1996; García Ferrando et al., 2002; Delgado, 2002; Martínez del Castillo et al., 2006). En este sentido, Fernández-Ballesteros, 1997; Fernández-Ballesteros, et al., 2004 y Fernández-Ballesteros, et al., 2007 analizan la percepción de la calidad de vida y el bienestar al recoger la importancia de las relaciones interpersonales propiciadas por la práctica deportiva.

Por tanto, ante esta creciente relevancia social del deporte y el importante papel que juegan las entidades deportivas en las relaciones sociales vinculadas con éste, resulta interesante establecer los perfiles socio-demográficos y socioeconómicos de aquellos que integran dichas entidades. Así, el objetivo principal de este trabajo es conocer los factores y las características más notables que condicionan el hecho de pertenecer a una entidad deportiva. Para realizar el estudio se utiliza un modelo de elección discreta binaria aplicado a los datos obtenidos de la encuesta "Hábitos Deportivos de los Españoles", realizada por el Centro de Investigaciones Sociológicas (CIS) en la primavera de 2005, por encargo del Consejo Superior de Deportes (CSD).

La investigación en el ámbito de las entidades deportivas se centra en la gestión y técnicas de su dirección y marketing (Aragón, 1992; Rodríguez, 2008). Además, por otra parte, también existen varios estudios que relacionan actividad deportiva y salud (Pons, 1998; Balaguer y Castillo, 2002; García et al., 2007; Sobejano et al., 2007; Arruza et al., 2008; Cancela y Pariente, 2008; Esnaola et al., 2011). Sin embargo, se hallan un menor número de estudios de carácter sociológico relacionados con el deporte. En este sentido, Buñuel (1994) apunta que la escasez de este tipo de estudios sociológicos "confirma la dicotomía clásica entre la mente y el cuerpo, separación que ha tenido como consecuencia un distanciamiento intelectual hacia todo lo que significa corporeidad". En España, García Ferrando analiza detalladamente en diversos estudios (García Ferrando, 1990, 1991 y 2001; García Ferrando et al., 2005) el aspecto social del deporte y los determinantes de la práctica deportiva, concluyendo que entre los más influyentes se encuentran el género, la edad, la renta, el nivel de estudios y las condiciones sociales. Por otra parte, Serrano (1998) clasifica los determinantes en culturales, de género y de tiempo libre, lo que refuerza la importancia de las condiciones sociales y culturales en la práctica deportiva. También Martínez del Castillo et al. (2006) recogen la existencia de relaciones significativas entre las condiciones de vida, los procesos de socialización y la actividad física en la vejez. En el caso de las mujeres, Puig y Soler (2004) indican que éstas al incor- 
porarse al mundo deportivo han creado su propia cultura deportiva sobre la que proyectan los valores adquiridos en el proceso de su socialización.

Las investigaciones que analizan los determinantes de la práctica deportiva se basan en diferentes enfoques. Por un lado, Schempp (1997) los clasifica en tres; empírico-analítico, histórico-hermenéutico y socio-crítico. Nuestro estudio se corresponde con el primero de ellos, empírico-analítico, y pretende definir las características del individuo que está interesado en pertenecer a una entidad deportiva. Por otro lado, otro aspecto fundamental que puede explicar el auge del sector deportivo en la sociedad moderna es la preocupación por el aspecto físico y el estado de salud y, a su vez, la conciencia de que la actividad deportiva favorece la consecución de ambos.

Tras la introducción, el presente trabajo se organiza en los siguientes apartados. La segunda sección presenta los datos y los modelos estimados, la tercera los resultados principales y, por último, la cuarta las conclusiones.

\section{MÉTODO}

\subsection{Datos}

La encuesta realizada por el Centro de Investigaciones Sociológicas (CIS) en la primavera de 2005, por encargo del Consejo Superior de Deportes (CSD), sobre los hábitos deportivos de los españoles trata de ofrecer una imagen, lo más precisa posible, de los comportamientos deportivos, así como de las actitudes, opiniones y valores en torno al deporte, de la población española de 15 a 74 años.

La información proporcionada es esencialmente de carácter socio-demográfico y socio-económico: edad, género, lugar de residencia, nivel de estudios, información de tipo laboral y una serie de variables sobre los hábitos deportivos de los individuos. Los datos de 2005 contienen información relativa a 8.170 personas adultas que conforman la muestra y el ámbito geográfico de la encuesta lo constituye el territorio del estado español.

En un principio se habían seleccionado 29 variables para ser incluidas en el modelo a estimar. En la Tabla 1 se presentan, clasificadas por grupos y subgrupos, las variables incluidas finalmente en el modelo. En la segunda y tercera columna constan la descripción y codificación de la encuesta original y en la quinta y sexta columna el nombre y los valores que toman estas variables tras su recodificación. Las variables seleccionadas son en su mayoría ficticias que recogen determinadas características de los individuos.

Del grupo de las variables socio-demográficas se incluyen aquellas como la edad, género, nivel de estudios y lugar de residencia que se definen a sí mismas por su denominación; y del grupo de las variables socio-laborales se incorporan las de situación laboral. Por otro lado, el grupo de hábitos deportivos contiene 
dos variables. La primera de ellas recoge si el individuo practica deporte y la segunda cuál es su apreciación de su forma física.

Tabla 1

Grupos de variables empleadas

\begin{tabular}{|c|c|c|c|c|}
\hline \multicolumn{5}{|c|}{ Variable dependiente } \\
\hline Grupo & Subgrupo & $\begin{array}{l}\text { Valores que toman las } \\
\text { variables en la encuesta }\end{array}$ & Variable & $\begin{array}{c}\text { Valores que toman las } \\
\text { variables en el modelo } \\
\text { estimado }\end{array}$ \\
\hline $\begin{array}{l}\text { Hábitos } \\
\text { Deportivos }\end{array}$ & $\begin{array}{l}\text { Pertenencia a } \\
\text { entidad } \\
\text { deportiva }\end{array}$ & $\begin{array}{l}\text { Es miembro de: Club privado, } \\
\text { gimnasio o similares, asociación } \\
\text { deportiva municipal, asociación } \\
\text { deportiva privada. Todos los } \\
\text { casos } 1=\mathrm{Sí}, 2=\text { No }\end{array}$ & $\begin{array}{l}\text { Pertenencia a } \\
\text { entidad } \\
\text { deportiva }\end{array}$ & $\begin{array}{l}1=\text { Pertenece a alguna } \\
\text { entidad deportiva, } 0=\text { Caso } \\
\text { contrario }\end{array}$ \\
\hline \multicolumn{5}{|c|}{ Variables explicativas } \\
\hline \multirow{6}{*}{$\begin{array}{l}\text { Socio- } \\
\text { demográficas }\end{array}$} & Edad & $\begin{array}{l}\text { De } 15 \text { a } 98=\text { Edad de } 15 \text { a } 98 \\
\text { años }\end{array}$ & Edad & De 15 a 98 años \\
\hline & Género & 1= Hombre, $2=$ Mujer & Género & 1= Hombre, $0=$ Mujer \\
\hline & \multirow{3}{*}{$\begin{array}{l}\text { Nivel de } \\
\text { estudios }\end{array}$} & $1=$ Sin estudios & Sin estudios & $\begin{array}{l}1=\text { Sin estudios, } 0=\text { Caso } \\
\text { contrario }\end{array}$ \\
\hline & & $\begin{array}{l}2=\text { Primaria, } 3=\text { Secundaria, } 4= \\
\text { Formación Profesional }\end{array}$ & $\begin{array}{l}\text { Estudios no } \\
\text { universitarios }\end{array}$ & $\begin{array}{l}1=\text { Estudios no } \\
\text { universitarios, } \\
0=\text { Caso contrario }\end{array}$ \\
\hline & & $\begin{array}{l}5=\text { Medios Universitarios, } 6= \\
\text { Universitarios Superiores }\end{array}$ & $\begin{array}{l}\text { Estudios no } \\
\text { universitarios }\end{array}$ & $\begin{array}{l}1=\text { Estudios universitarios, } \\
0=\text { Caso contrario }\end{array}$ \\
\hline & Residencia & $\begin{array}{l}\text { De } 1 \text { a } 19 \text { para todas las } \\
\text { comunidades autónomas }\end{array}$ & Nombre CA & $\begin{array}{l}1=\text { Comunidad de } \\
\text { residencia, } \\
0=\text { Caso contrario }\end{array}$ \\
\hline \multirow{4}{*}{$\begin{array}{l}\text { Socio- } \\
\text { laborales }\end{array}$} & \multirow{4}{*}{$\begin{array}{l}\text { Situación de } \\
\text { actividad }\end{array}$} & $1=$ Trabaja & Trabaja & $\begin{array}{l}1=\text { Trabaja, } 0=\text { Caso } \\
\text { contrario }\end{array}$ \\
\hline & & $\begin{array}{l}\text { Jubilado que } 2=\text { ha trabajado, } 3= \\
\text { no ha trabajado }\end{array}$ & Jubilado & $\begin{array}{l}1=\text { Jubilado, } 0=\text { Caso } \\
\text { contrario }\end{array}$ \\
\hline & & $\begin{array}{l}\text { Parado que } 4=\text { ha trabajado, } 5= \\
\text { busca } 1^{\text {er }} \text { empleo }\end{array}$ & Desempleado & $\begin{array}{l}1=\text { Desempleado, } 0=\text { Caso } \\
\text { contrario }\end{array}$ \\
\hline & & $\begin{array}{l}\text { 6= Estudiante, } 7=\text { Trabajo } \\
\text { doméstico, } 8=\text { Otra sit. }\end{array}$ & Otra situación & $\begin{array}{l}1=\text { Otra situación, } 0=\text { Caso } \\
\text { contrario }\end{array}$ \\
\hline \multirow{5}{*}{$\begin{array}{l}\text { Hábitos } \\
\text { Deportivos }\end{array}$} & \multirow{2}{*}{ Forma física } & $\begin{array}{l}\text { Apreciación forma física 1= } \\
\text { Excelente, } 2=\text { Buena }\end{array}$ & $\begin{array}{l}\text { Forma física } \\
\text { buena }\end{array}$ & $\begin{array}{l}1=\text { Forma física buena, } \\
0=\text { Caso contrario }\end{array}$ \\
\hline & & $\begin{array}{l}3=\text { Aceptable, } 4=\text { Deficiente }, 5= \\
\text { Muy mala }\end{array}$ & $\begin{array}{l}\text { Forma física } \\
\text { mala }\end{array}$ & $\begin{array}{l}1=\text { Forma física mala, } \\
0=\text { Caso contrario }\end{array}$ \\
\hline & \multirow{3}{*}{$\begin{array}{l}\text { Práctica } \\
\text { deportiva }\end{array}$} & $\begin{array}{l}\text { 1= Practica un deporte, 2= } \\
\text { Practica Varios }\end{array}$ & Practica deporte & $\begin{array}{l}1=\text { Practica deporte } \\
0=\text { Caso contrario }\end{array}$ \\
\hline & & $3=$ No practica ninguno & $\begin{array}{l}\text { No practica } \\
\text { deporte }\end{array}$ & $\begin{array}{l}1=\text { No practica deporte } \\
0=\text { Caso contrario }\end{array}$ \\
\hline & & $\begin{array}{l}3=\text { Aceptable, } 4=\text { Deficiente, } 5= \\
\text { Muy mala }\end{array}$ & $\begin{array}{l}\text { Forma física } \\
\text { mala }\end{array}$ & $\begin{array}{l}1=\text { Forma física mala, } \\
0=\text { Caso contrario }\end{array}$ \\
\hline
\end{tabular}

Fuente: Elaboración propia.

En un principio se habían extraído también otros grupos de variables como, por ejemplo, las relacionadas con la actividad física del encuestado en su trabajo o variables que recogen el grado de aceptación que presenta el sujeto frente a 
distintas afirmaciones relacionadas con el deporte tales como: "El deporte te permite estar en forma", "El deporte es fuente de salud" o "El deporte es como una válvula de escape para liberar saludablemente la agresividad y el mal humor". Además, se había considerado también la inclusión de variables relacionadas con la medicina-dietética deportiva que reflejan si el encuestado usa actualmente o ha usado con anterioridad algún medicamento o suplemento dietético destinado a mejorar la práctica de la actividad física o deportiva. No obstante, las variables de estos tres últimos grupos no han sido finalmente incluidas en el modelo por no resultar significativas.

Dentro del epígrafe Pertenencia a entidad deportiva se agrupan variables como la pertenencia a distintas entidades deportivas, los motivos de pertenencia a un club o asociación y los motivos de abandono. Las variables que indican si un individuo pertenece a un club privado, gimnasio o similares, asociación deportiva municipal o asociación deportiva privada se han recodificado y unificado en una única variable ficticia (Pertenencia a entidad deportiva) que toma valor 1 si el individuo pertenece a alguna entidad deportiva y valor 0 en caso contrario y representa la variable dependiente del modelo estimado. La definición de esta variable considera la pertenencia a las entidades deportivas públicas y privadas, sin establecer distinción entre ellas. Tras realizar esta restricción y depurar las observaciones ausentes o incompletas, al final se dispone de una muestra de 3.490 individuos. De éstos, el $61 \%$ pertenece a alguna entidad deportiva.

\subsection{Metodología}

La teoría de clubes es, a parte de los clubes deportivos, aplicable a estudios de áreas recreativas, autopistas o sistemas de comunicación. Sandler y Tschirhart (1997) en su trabajo que revisa esta teoría presentan tres modelos básicos y describen sus posibles extensiones incluyendo socios heterogéneos, costes de transacción o información asimétrica. Estos modelos teóricos proporcionan niveles óptimos de variables como el número de socios o coste de pertenencia al club. Sin embargo, Sandler y Tschirhart (1997) destacan la falta de aplicaciones empíricas necesarias para el apoyo de los resultados teóricos.

El presente trabajo aplicado pertenece a la literatura de los estudios empíricos de la demanda de deporte sin disponer de los gastos concretos de los individuos en este concepto. El modelo de elección discreta utilizado está basado en la clásica teoría de utilidad aleatoria (McFadden, 1981) donde un individuo elije entre dos alternativas y la utilidad de la alternativa $j$ se define como

$$
U_{j}=\beta^{\prime} X_{j}+\varepsilon_{j},
$$


donde $X_{j}$ es un vector de características observadas del individuo $j, \beta$ es un vector de parámetros desconocidos y $\varepsilon_{j}$ es un error aleatorio que recoge los atributos no observados del individuo y de la alternativa. Dependiendo de los supuestos, la ecuación [1] puede llevar a diferentes modelos de elección discreta. En este trabajo se aplica el modelo logit binario teniendo en cuenta la posible heteroscedasticidad del error aleatorio (Long, 1997; Greene, 2011). Dado que, en nuestro caso, la variable analizada es:

$$
Y_{i}=\left\{\begin{array}{l}
1 \text { si el individuo i pertenece a una entidad depotiva } \\
0 \quad \text { en caso contrario. }
\end{array}\right.
$$

la utilidad subyacente analizada es la utilidad de la pertenencia a una entidad deportiva. La elección de las características $X_{j}$ en [1] se basa por tanto en la literatura de la demanda deportiva. El supuesto aplicado sobre el comportamiento de los errores $\varepsilon_{j}$ de la ecuación de la utilidad aleatoria subyacente a un modelo logit es

$$
\operatorname{Var}\left(\varepsilon_{i}\right)=\sigma_{i}^{2}=\exp \left(Z_{i}^{\prime} \gamma\right)^{2},
$$

donde $Z_{i}$ contiene observaciones de variables que puedan explicar una posible heteroscedasticidad del error de la variable latente y $\gamma$ son parámetros a estimar. La probabilidad de que $Y_{i}=1$ se define en este caso como:

$$
\operatorname{Pr}\left(Y_{i}=1\right)=\Lambda\left[\frac{X_{i}^{\prime} \beta}{\exp \left(Z_{i}^{\prime} \gamma\right)}\right] .
$$

Dado que casi todas menos una de las variables explicativas incluidas en el modelo son variables ficticias, la expresión a utilizar para analizar su efecto sobre la $\operatorname{Pr}\left(Y_{i}=1\right)$ es,

$$
\operatorname{Pr}\left(Y_{i}=1 \mid \widetilde{X}_{i}^{(1)}, \widetilde{Z}_{i}\right)-\operatorname{Pr}\left(Y_{i}=1 \mid \tilde{X}_{i}^{(0)}, \widetilde{Z}_{i}\right)=\Lambda\left(\frac{\widetilde{X}_{i}^{(1)} \beta}{\exp \left(\widetilde{Z}_{i} \gamma\right)}\right)-\Lambda\left(\frac{\widetilde{X}_{i}^{(0)} \beta}{\exp \left(\widetilde{Z}_{i} \gamma\right)}\right)
$$

donde el valor de la variable ficticia cuyo efecto se analiza está fijado en 1 en el vector $\widetilde{X}_{i}^{(1)}$ y 0 en el vector $\widetilde{X}_{i}^{(0)}$. Todas las demás variables en $\widetilde{Z}_{i}, \widetilde{X}_{i}^{(0)}$ y $\widetilde{X}_{i}^{(1)}$ están fijadas en su valor medio (si son variables cuantitativas) y 0 ó 1 (si son variables ficticias). El valor 0 ó 1 se elige de tal forma que la interpretación sea realista. 
Lo que se refiere a la elección de las características $X_{j}$, Pawlowski y Breuer (2011) observan los gastos en servicios deportivos y recreacionales en Alemania utilizando un modelo de demanda basado en el marco teórico de la maximización de la utilidad. Las características de los individuos incluidos en su análisis incluyen las variables socio-demográficas que generalmente coinciden con las utilizadas en el presente estudio. Una de las clases de gastos que comparan es la correspondiente a los gastos de cuotas en clubes deportivos, por lo que tiene cierta similitud con este trabajo. En relación a otras investigaciones que examinan el efecto de las variables socio-demográficas en un marco similar se puede mencionar Lera-López y Rapún-Gárate (2007) que analizan la práctica deportiva y gastos de consumo en España; Breuer y Schlesinger (2006) que observan el consumo y demanda de bienes deportivos en Alemania; Taks, et al. (1999) que se centran en los gastos deportivos en Bélgica; o Breuer et al. (2010) que consideran el nivel de actividad física de los individuos y sus gastos deportivos.

Por último, señalamos como importante, que la metodología de un modelo heteroscedástico de elección binaria aplicada es relativamente simple y no posee un carácter novedoso en el campo del deporte. Sin embargo, dadas las limitaciones de los datos y el número de observaciones finalmente disponible, su simplicidad puede proporcionar cierta robustez difícil de conseguir con otros enfoques basados en datos que contengan más información. De estos enfoques se puede mencionar p.ej. los modelos logit mixtos utilizados desde hace varios años de forma existosa en el campo del transporte, la salud o el medioambiente. Los artículos de Barros et al. (2007) y Frick (2010) pueden ser citados como aplicaciones de estos modelos en la literatura del deporte.

\section{RESULTADOS}

En la Tabla 2 se presenta el porcentaje de individuos pertenecientes a cada categoría de las variables ficticias y de manera separada los estadísticos descriptivos de la única variable continua del modelo (Edad). Esta tabla recoge, además, el indicador GVIF (Generalized variance inflation factor) que se utiliza para la detección de multicolinealidad (Fox y Monette, 1992).

La gran mayoría de las variables explicativas presentan un valor GVIF muy bajo, indicando poca correlación entre ellas. Sólo las variables relacionadas con el nivel de estudios ofrecen valores superiores a 10, lo que muestra un cierto grado de multicolinealidad (Myers, 1990). Sin embargo, se opta por mantener estas variables en el modelo por su relevancia esperada, teniendo en cuenta que una posible multicolinealidad puede aumentar el errores estándar de los parámetros. 
Tabla 2

Variables incluidas en la estimación y su definición

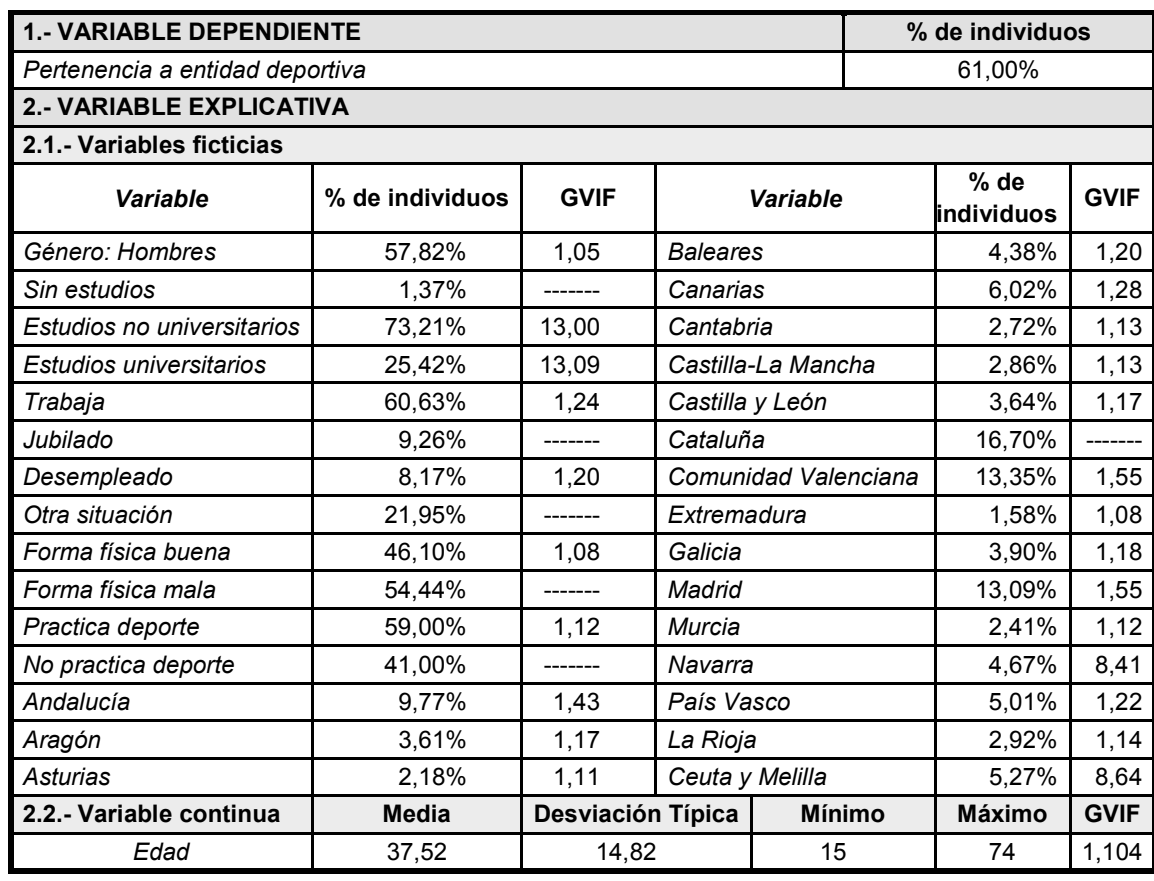

Fuente: Elaboración propia.

La Tabla 3 presenta la estimación del modelo logit sin y con heteroscedasticidad e incluye los coeficientes estimados, el error estándar y los valores $p$ de contrastes de significatividad individual de cada parámetro. Ambos modelos fueron estimados por el método de máxima verosimilitud.

La primera columna contiene el nombre de la variable explicativa, las siguientes tres columnas los resultados obtenidos para un logit binario y las tres últimas corresponden a un logit binario heteroscedástico. Una vez tratada la heteroscedasticidad de los errores, todas las variables explicativas son significativas a nivel de significatividad menor que 5\%, excepto algunas variables ficticias de siete comunidades autónomas.

La Tabla 3 se completa con indicadores de la bondad de ajuste. Se puede observar una mejora en el ajuste del modelo logit heteroscedástico, ya que el valor de log-verosimilitud aumenta y el indicador de bondad de ajuste AIC disminuye. Además la variable Edad incluida en el vector $Z_{i}$ (ecuación [3]), que indica la existencia de heteroscedasticidad, es significativa individualmente a nivel menor que $1 \%$. 
La interpretación de los resultados se basa por tanto en los signos de los coeficientes estimados de la quinta columna de la Tabla 3 y en los efectos de un cambio unitario en las variables explicativas sobre la probabilidad de pertenecer a una entidad deportiva que se presentan en la Tabla 4. En nuestro caso, este cambio unitario representa la variación de tener a no tener cierta característica, ya que las variables incluidas en la tabla son ficticias.

Tabla 3

Estimación de la variable pertenencia a una entidad deportiva

\begin{tabular}{|c|c|c|c|c|c|c|c|c|}
\hline \multicolumn{9}{|c|}{ Método de estimación: máxima verosimilitud } \\
\hline \multicolumn{9}{|c|}{ Número de observaciones: 3.490} \\
\hline \multirow[b]{2}{*}{ Variable } & \multicolumn{4}{|c|}{ Logit binario } & \multicolumn{4}{|c|}{ Logit binario-Heteroscedasticidad } \\
\hline & \multirow{2}{*}{\begin{tabular}{|c|} 
Coeficiente \\
$-2,79$
\end{tabular}} & \multirow{2}{*}{\begin{tabular}{|c|} 
Error Est. \\
0,467
\end{tabular}} & \multicolumn{2}{|c|}{ Valor $p$} & \multirow{2}{*}{\begin{tabular}{|c|} 
Coeficiente \\
$-5,708$
\end{tabular}} & \multirow{2}{*}{$\begin{array}{c}\text { Error Est. } \\
1,257\end{array}$} & \multicolumn{2}{|c|}{ Valor $p$} \\
\hline Constante & & & $<0,010$ & *** & & & $<0,010$ & *** \\
\hline Edad & 0,004 & 0,003 & 0,121 & & 0,017 & 0,006 & $<0,010$ & *** \\
\hline Género & $-0,250$ & 0,087 & $<0,010$ & *** & $-0,503$ & 0,162 & $<0,010$ & $* \star *$ \\
\hline Estudios no universitarios & 1,731 & 0,414 & $<0,010$ & *** & 3,607 & 1,040 & $<0,010$ & *** \\
\hline Estudios universitarios & 1,808 & 0,420 & $<0,010$ & *** & 3,753 & 1,057 & $<0,010$ & *** \\
\hline Trabaja & $-0,243$ & 0,968 & 0,012 & ** & $-0,364$ & 0,164 & 0,027 & ** \\
\hline Desempleado & $-0,663$ & 0,164 & $<0,010$ & $* * *$ & $-1,045$ & 0,282 & $<0,010$ & *** \\
\hline Forma física buena & 0,303 & 0,866 & $<0,010$ & $* * *$ & 0,475 & 0,150 & $<0,010$ & *** \\
\hline Practica deporte & 2,426 & 0,898 & $<0,010$ & *** & 4,032 & 0,408 & $<0,010$ & $* * *$ \\
\hline Andalucía & 0,268 & 0,165 & 0,106 & & 0,340 & 0,270 & 0,208 & \\
\hline Aragón & 0,653 & 0,249 & $<0,010$ & *** & 1,060 & 0,429 & 0,135 & ** \\
\hline Asturias & 0,594 & 0,316 & 0,060 & * & 1,026 & 0,536 & 0,557 & * \\
\hline Baleares & 0,036 & 0,218 & 0,868 & & $-0,110$ & 0,361 & 0,760 & \\
\hline Canarias & 0,525 & 0,198 & $<0,010$ & $* * *$ & 0,800 & 0,339 & 0,018 & ** \\
\hline Cantabria & 0,838 & 0,277 & $<0,010$ & $* \star \star$ & 1,264 & 0,448 & $<0,010$ & *** \\
\hline Castilla-La Mancha & 0,760 & 0,267 & $<0,010$ & *** & 1,110 & 0,432 & 0,010 & ** \\
\hline Castilla y León & 0,805 & 0,247 & $<0,010$ & *** & 1,294 & 0,398 & $<0,010$ & *** \\
\hline Comunidad Valenciana & $-0,216$ & 0,149 & 0,147 & & $-0,387$ & 0,273 & 0,156 & \\
\hline Extremadura & 0,150 & 0,349 & 0,668 & & 0,052 & 0,536 & 0,922 & \\
\hline Galicia & 0,561 & 0,240 & 0,019 & ** & 0,848 & 0,371 & 0,022 & ** \\
\hline Madrid & $-0,034$ & 0,151 & 0,823 & & $-0,085$ & 0,251 & 0,736 & \\
\hline Murcia & $-0,189$ & 0,281 & 0,502 & & $-0,260$ & 0,485 & 0,592 & \\
\hline Navarra & 0,178 & 0,601 & 0,767 & & 0,109 & 0,785 & 0,890 & \\
\hline País Vasco & 0,552 & 0,216 & 0,105 & ** & 0,813 & 0,360 & 0,024 & ** \\
\hline La Rioja & 1,024 & 0,299 & $<0,010$ & $* * *$ & 1,769 & 0,575 & $<0,010$ & $* * \star$ \\
\hline Ceuta y Melilla & 0,780 & 0,572 & 0,173 & & 1,499 & 0,740 & 0,043 & ** \\
\hline \multicolumn{5}{|c|}{ Variables causantes de heteroscedasticidad } & Coeficiente & Error Est. & \multicolumn{2}{|c|}{ Valor $p$} \\
\hline Edad & & & & & 0,135 & 0,003 & $<0,010$ & *** \\
\hline \multicolumn{9}{|c|}{ 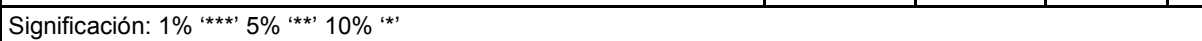 } \\
\hline \multicolumn{3}{|c|}{ Indicadores de bondad del ajuste } & \multicolumn{2}{|c|}{ Valor } & & & \multicolumn{2}{|c|}{ Valor } \\
\hline \multicolumn{3}{|l|}{ Log-verosimilitud } & \multicolumn{2}{|c|}{$-1.760,94$} & & & \multicolumn{2}{|c|}{$-1.747,24$} \\
\hline \multicolumn{3}{|l|}{$\mathrm{AIC}$} & \multicolumn{2}{|c|}{1,024} & & & \multicolumn{2}{|c|}{1,017} \\
\hline
\end{tabular}

Fuente: Elaboración propia. 
Tabla 4

Efectos sobre la probabilidad de pertenencia a entidad deportiva

\begin{tabular}{|l|l|c|}
\hline Característica base & Cambio en la característica base & $\begin{array}{c}\text { Efecto del } \\
\text { cambio sobre la } \\
\text { probabilidad }\end{array}$ \\
\hline Género: Mujer & Género: Hombre & $-0,0114$ \\
\hline \multirow{2}{*}{ Residencia: Cataluña } & Residencia: Castilla-La Mancha & 0,0394 \\
\cline { 2 - 3 } & Residencia: La Rioja & 0,0756 \\
\hline \multirow{2}{*}{ Nivel de estudios: Sin estudios } & Nivel de estudios: No universitarios & 0,2490 \\
\cline { 2 - 3 } & Nivel de estudios: Universitarios & 0,2676 \\
\hline \multirow{2}{*}{ Situación de actividad: } & Trabaja & $-0,0085$ \\
\cline { 2 - 3 } Otra situación & Desempleado & $-0,0205$ \\
\hline Forma física percibida: Mala & Forma física percibida: Buena & 0,0141 \\
\hline No practica deporte & Practica deporte & 0,3049 \\
\hline
\end{tabular}

Fuente: Elaboración propia.

Para poder calcular estos efectos se debe definir un individuo base que servirá de referencia en las comparaciones. Para este individuo (definido por las características de la primera columna de la Tabla 4) se fija la edad en su media muestral y todas las demás variables, por ser ficticias, en el valor cero o uno. Por tanto, la Tabla 4 presenta los cambios en la probabilidad de pertenencia a entidad deportiva de un individuo que posee o no cierta característica diferente con respecto al individuo base calculados según la ecuación [5].

Los signos de los coeficientes estimados de la Tabla 3 indican si el efecto de una variable explicativa sobre esta probabilidad es positivo o negativo. En general, todos los coeficientes estimados presentan signos que coinciden a priori con las hipótesis.

La edad resulta ser una variable significativa al $1 \%$ sólo cuando se realiza el tratamiento de la heteroscedasticidad. Su efecto no es estrictamente positivo, ya que es la variable causante de la heteroscedasticidad y aparece tanto entre las variables $X_{i}$ como entre las $Z_{i}$ de la ecuación [4]. En la Figura 1 se puede observar su efecto cambiante sobre la probabilidad de pertenecer a una entidad deportiva, dependiendo de que el individuo practique o no deporte. Para esta comparación se selecciona un sujeto con alta probabilidad de pertenencia a una entidad deportiva (mujer universitaria que trabaja, residente en La Rioja y que percibe que su forma física es buena).

Es lógico que un individuo que no practica deporte y de alrededor de 40 años posea una mayor probabilidad de pertenecer a una entidad deportiva que un menor de 16 años con el resto de características iguales, pero carece de sentido que ese individuo de 40 años tenga menor probabilidad que otro que tiene más de 80 años. En efecto, al tratar la heteroscedasticidad, los resultados obtenidos 
son más acordes con las hipótesis a priori. La probabilidad aumenta para individuos jóvenes pero a partir de cierta edad esta probabilidad disminuye. Por otro lado, fijándonos sólo en el signo positivo del coeficiente de la variable Edad en el término de la heteroscedasticidad, se puede concluir que individuos mayores presentan mayor dispersión a la hora de decidir si asociarse o no a una entidad deportiva.

Figura 1

Probabilidad estimada de pertenencia a una entidad deportiva.

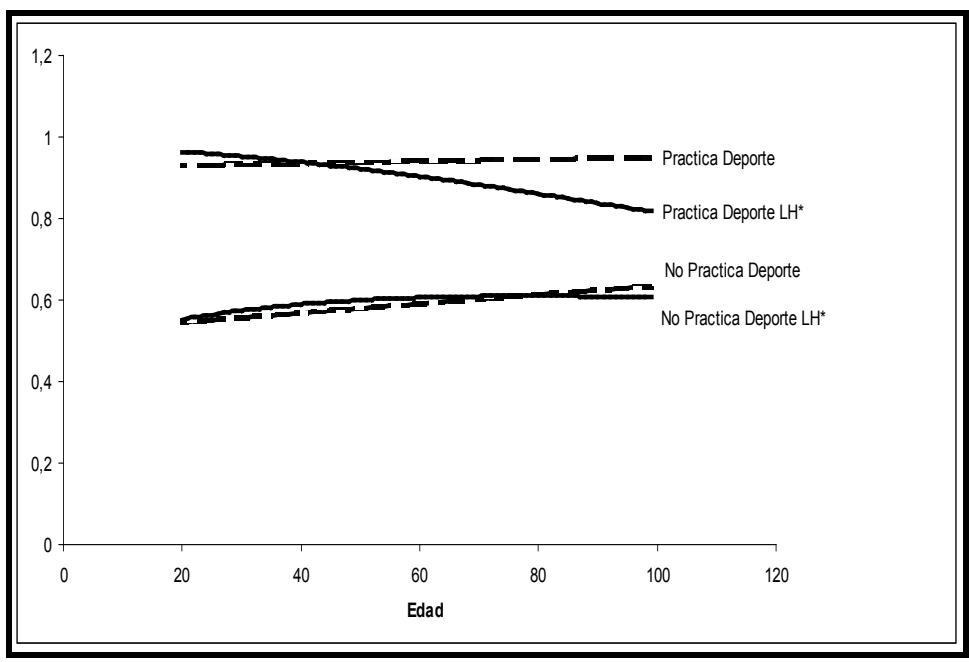

(*LH: Logit heteroscedástico)

Fuente: Elaboración propia.

Pawlowski y Breuer (2011) analizan el efecto de las variables como edad, estado civil, estructura de la unidad familiar y tipo de zona de residencia sobre los gastos deportivos y recreacionales, encontrando un resultado similar respecto a la edad. En concreto, sus conclusiones indican un efecto significativo de la edad sobre la probabilidad de pertenecer a un club deportivo. El efecto encontrado es, en su caso, también cóncavo ya que, en su modelo Heckit estimado, la variable edad tiene signo positivo y el cuadrado de la misma variable signo negativo. Según este resultado, la probabilidad es máxima para unidad familiar donde el cabeza de familia tiene edad mediana.

Serrano (1999) examina la relación entre la práctica deportiva y las audiencias de eventos deportivos centrándose en la eficiencia de la promoción deportiva a través de la organización de eventos deportivos y su retransmisión. Concluye, al igual que nuestro estudio, que a partir de cierta edad y a medida que ésta aumenta se produce una reducción en la práctica deportiva aunque no 
ocurre lo mismo con las audiencias. Por ello, interpreta que en edades avanzadas aparecen frenos psicológicos y barreras sociales que provocan la disminución de la práctica deportiva, hecho que no sucede con las audiencias deportivas. En la misma línea, Collins (2003) indica que las características y el entorno social en el que se desenvuelven las personas mayores pueden convertirse en un obstáculo para el acceso de éstos a la práctica deportiva.

En Campos et al. (2005, 2010 y 2011) y en Jiménez-Beatty (2002) se muestra que las personas mayores demandan monitores deportivos y que es en las entidades deportivas y residencias donde se genera la mayor solicitud. Concretamente, Campos et al. (2010) encuentran que en el 87,5\% de las entidades deportivas existe presencia de monitores en la actividad física y deporte para mayores, frente al 4,8\% de actividades deportivas de autoorganización que cuentan con estos profesionales. Con ello, se puede entender que aunque para las personas mayores la probabilidad de pertenecer a una entidad deportiva disminuye, ésta sigue siendo considerable. De este modo, en varios estudios se recoge que, pese a que las personas mayores en comparación con otros grupos de edad son las que menos actividad física realizan, los cambios sociales, económicos y culturales que se están produciendo en las últimas décadas fomentan una mayor práctica deportiva entre las personas de edad avanzada (Bazo, 1996; Jamet, 1998; García Ferrando, 2001; Mosquera y Puig, 2002; Campos et al., 2010).

La variable que recoge el efecto del género (Género) muestra una relación negativa con la probabilidad de pertenecer a una entidad deportiva. Dado que esta variable está definida como cero para mujeres y uno para hombres, las mujeres tienen mayor probabilidad de pertenecer a una entidad deportiva que los hombres. Es un resultado sorprendente dado el importante peso específico que tiene el deporte masculino. Así lo recoge por ejemplo García et al. (2007) indicando que el mundo del deporte sigue siendo mayoritariamente masculino debido: al dominio y el poder que la sociedad todavía otorga a los hombres, a que el modelo de educación física fomenta la competitividad, la fuerza y la agresividad frente a otros valores y a que los medios de comunicación continúan ofreciendo mayor repercusión al deporte masculino.

La explicación de que las mujeres tengan mayor probabilidad de pertenecer a una entidad deportiva podría encontrarse en que, por tradición, la mujer ha estado más preocupada por su físico y ha procurado cuidarlo con actividades que habitualmente se desarrollan en entidades deportivas. Así lo entienden Buñuel (1994) y García et al. (2007) que concluyen que la actividad física en las mujeres en la actualidad está encaminada hacia el deporte-salud y el deporte-recreativo. Es cierto que el hombre es más deportista, pero en rasgos generales disfruta de actividades al aire libre como puede ser la carrera a pie, el ciclismo o los deportes de aventura. Es menor el número de hombres a los que les gusta 
practicar deportes sometidos a unos horarios y bajo la supervisión que suele existir en las entidades deportivas.

En otras palabras, puede que en general las mujeres no realicen un gran esfuerzo físico en su actividad deportiva, pero poseen la suficiente constancia como para que les compense abonar la cuota mensual de una entidad deportiva y además suelen sentirse más seguras si su actividad está dirigida por un profesional. En este sentido, el estudio de Cancela y Pariente (2008) indica que las mujeres realizan con mayor probabilidad actividad física de forma regular (en un club deportivo o en un gimnasio) y los hombres destacan en el volumen de tiempo dedicado al deporte. También Pavón y Moreno (2008) estudian las diferencias por género en los estudiantes de la Universidad de Murcia ante la práctica deportiva. Los autores concluyen que los hombres están principalmente interesados en el aspecto competitivo de la actividad física y que a las mujeres les preocupa más la repercusión de ésta sobre la salud.

En la Tabla 4 se puede observar que un hombre, con las características definidas en la primera columna, tiene un $1,14 \%$ menos de probabilidad de pertenecer a una entidad deportiva que una mujer con las mismas particularidades. La diferencia no es excesiva porque, además del elevado número de hombres que están abonados a los gimnasios, existen deportes practicados en su mayoría por varones en los que es necesaria la pertenencia a una entidad deportiva.

El lugar de residencia ha sido estudiado para todas las comunidades autónomas siendo la categoría de referencia la Comunidad Autónoma de Cataluña. La variable ficticia del lugar de residencia en la mayoría de las comunidades autónomas ha resultado ser una variable significativa. A continuación se analiza el caso de la Comunidad Autónoma de La Rioja porque, a pesar de ser una de las comunidades autónomas más pequeñas, se encuentra entre las de mayor renta per capita y el caso de la Comunidad Autónoma de Castilla-La Mancha que, por el contrario, se halla entre las de menor renta per capita. Ante este hecho, residir en La Rioja o Castilla-La Mancha presenta una relación positiva con la probabilidad de pertenecer a una entidad deportiva frente a vivir en Cataluña. Para analizar este resultado es importante recordar que se ha considerado la pertenencia a las entidades deportivas tanto privadas como públicas.

En Cataluña se encuentran los clubes privados más importantes del Estado, pero a ellos accede sólo una pequeña parte de la población. Sin embargo, en cuanto a instalaciones deportivas municipales se refiere, como entidades deportivas que acogen a la gran mayoría de la población, La Rioja y Castilla-La Mancha presentan una oferta más numerosa en términos relativos. Así lo demuestra el 40,1\% de la población de Castilla-La Mancha y el 39,4\% de la población de La Rioja que opina que existen instalaciones deportivas suficientes, frente al $37 \%$ de la población de Cataluña. 
Por otro lado, el hecho de residir en grandes ciudades en las que cualquier desplazamiento supone una importante inversión de tiempo podría implicar que los individuos que desean hacer deporte opten por realizar actividades para las que no es necesario desplazarse hasta una entidad deportiva. García et al. (2007) incluso afirman que "la urbanización nos lleva al sedentarismo y al estrés". También es importante destacar que la cultura deportiva y el porcentaje relativo de población que practica deporte en La Rioja son mayores. En la encuesta de "Hábitos Deportivos de los Españoles" que se está estudiando, se incluye una pregunta sobre el interés que tienen los encuestados por el deporte y su nivel de práctica. De los residentes en Cataluña el $16,1 \%$ de la población declaró que le interesaba el deporte y lo practicaba "suficiente" mientras que en La Rioja el porcentaje fue del $21,1 \%$. Además, con respecto a la población que nunca había pertenecido a una entidad deportiva, al $25,7 \%$ en Castilla-La Mancha y al $18,6 \%$ en La Rioja, les gustaría formar parte de alguna en el futuro. En Cataluña sólo el $17,4 \%$ de dicha población realizaba la misma afirmación. Por tanto, los resultados obtenidos en este estudio corroboran estos datos ya que en la Tabla 4 se observa cómo la probabilidad de pertenecer a una entidad deportiva para un individuo de las mismas características es, con respecto a los residentes en Cataluña, un 7,56\% mayor para los residentes de la Comunidad Autónoma de La Rioja y un 3,94\% mayor para los residentes de la Comunidad Autónoma de Castilla-La Mancha.

El nivel de estudios se ha dividido en dos grupos: no universitarios (Estudios no universitarios) y universitarios (Estudios universitarios) frente a los individuos sin estudios. Ambos grupos se conforman como variable significativa y muestran una relación positiva con la probabilidad de pertenecer a una entidad deportiva. Los individuos con mayor probabilidad son aquellos que poseen estudios universitarios. La probabilidad aumenta en un $26,76 \%$ si el individuo tiene estudios universitarios y en un $24,9 \%$ si dispone de estudios no universitarios frente a los sujetos sin estudios. Martínez del Castillo et al. (2006) también encuentran relaciones significativas entre el nivel de estudios y la actividad física y concluyen que cuanto mayor es el capital económico, cultural y social que posee un individuo, mayor es la probabilidad de que éste "participe en procesos de socialización en la actividad física".

Las situaciones laborales cuando la persona desempeña un trabajo (Trabaja) y se encuentra desempleado (Desempleado) presentan relación negativa con la probabilidad de pertenecer a una entidad deportiva. Se podría entender que ello es debido en el primer caso por falta de tiempo y en el segundo por menor poder adquisitivo. Con respecto a la falta de tiempo, García et al. (2007) explican que el incremento de horas trabajadas y de las responsabilidades familiares favorecen el sedentarismo de las mujeres. También Martínez del Castillo et al. (2006) identifican como principales causantes del abandono de la práctica deportiva: el incremento de las obligaciones y responsabilidades como puede ser el trabajo 
fuera y dentro del hogar y la falta de oportunidades marcada por la posición social o económica.

Los individuos con las mismas características del individuo base, pero que forman parte de la población activa (trabajadores y desempleados), tienen menor probabilidad de pertenecer a una entidad deportiva que aquellos que se encuentran en otra situación (jubilados, amas de casa, estudiantes y otros). La probabilidad disminuye aún más cuando se considera a los desempleados que cuando la comparación se realiza con los trabajadores. Este resultado demuestra que, para asociarse a una entidad deportiva, tendrán mayores reticencias aquellos individuos desempleados que otros que, por motivos laborales, carezcan de tiempo para acudir a ellas.

La variable que informa sobre si el individuo entiende que su forma física es buena (Forma física buena) es significativa al 1\% y presenta una relación positiva con la pertenencia a una entidad deportiva. Si el sujeto percibe que su forma fisica es buena, la probabilidad de pertenecer a una entidad deportiva aumenta en un $1,41 \%$. En este sentido, es lógico pensar que una persona que sienta que su forma física es buena decida pertenecer a una asociación deportiva en la que disfrutar de las distintas actividades que su condición física le permite y además se marque el objetivo de mantenerla por los beneficios que conlleva para su salud. Efectivamente, Arruza et al. (2008) demuestran que el estado de ánimo depende de la percepción del estado físico. En el trabajo de García et al. (2007), la práctica deportiva presenta relación positiva con cinco de los siete índices que se utilizan en su encuesta para definir la valoración de la salud, constatando así la interrelación que se produce entre percepción de salud y actividad física. También Pons (1998) y Balaguer y Castillo (2002) recogen en sus estudios la importancia de realizar actividad física con regularidad para poseer un buen estado de salud y menos probabilidades de enfermar.

Respecto a la variable que indica si el individuo practica alguno o varios deportes (Practica deporte), resulta ser significativa al 1\% y muestra una relación positiva con la pertenencia a una entidad deportiva. Aunque el individuo puede practicar por su cuenta una gran cantidad de deportes, existen otros muchos en los que es necesaria la estructura de una entidad deportiva. Por tanto, resulta coherente pensar que el hecho de practicar deporte influirá de manera positiva en el interés del individuo por pertenecer a una entidad deportiva.

Como se observa en la Tabla 4, la probabilidad de pertenecer a una entidad deportiva para un individuo que practica algún deporte es prácticamente un $31 \%$ más alta que para los individuos que no lo hacen. También, según los resultados de la encuesta "Hábitos Deportivos de los Españoles", el 30,28\% de los individuos que no practican deporte pertenecen a una entidad deportiva. En ese caso, se podría pensar en aquellos que en su día fueron deportistas y mantienen su vinculación a la entidad por tradición o porque la entidad deportiva pueda tener 
además un carácter social. Otra explicación menos deportiva la encontramos en todas aquellas personas que se asocian a una entidad deportiva para acompañar a sus hijos a cursillos de diferentes actividades deportivas, por ejemplo.

\section{CONCLUSIONES}

En este trabajo se estudian los factores y características más relevantes que condicionan el hecho de pertenecer a una entidad deportiva mediante un modelo logit binario heteroscedástico. La edad es la variable que recoge el efecto de la heteroscedasticidad, mostrando que individuos con edades avanzadas deciden la pertenencia a entidad deportiva con mayor variabilidad que sujetos jóvenes con el resto de características idénticas.

Para determinar la demanda de pertenencia a una entidad deportiva, no han resultado significativas diversas variables socio-económicas. Evidentemente, el hecho de que se hayan considerado todo tipo de entidades deportivas, incluidas las públicas, ha influido en este resultado. Parece lógico pensar que las variables resultarían significativas si el estudio se centrase en entidades deportivas privadas que son aquellas en las que, por norma general, se concentran socios con un nivel de renta medio-alto. En este sentido, Campos et al. (2010) que analizan la existencia de monitores deportivos en la actividad física y deportiva de las personas mayores no encuentran, tal y como sucede en nuestro estudio, diferencias entre las clases sociales ni entre las personas con ingresos altos y bajos.

Sin embargo, existen estudios que presentan a la clase social baja (Martínez del Castillo et al., 2006; González et al., 2008) y a los ingresos bajos como posibles barreras de acceso a la actividad deportiva. Los ingresos son analizados para el caso español en los trabajos de Martínez del Castillo et al. (2006) y Del Hierro et al. (2008) y, para el estadounidense, en McGuire (1985), Shephard (1994) y Collins (2003). Por tanto, el efecto de la renta sobre la actividad deportiva se trata de un interesante tema a analizar mediante encuestas y bases de datos alternativas en futuras investigaciones que deberían explicar las contradicciones encontradas.

La variable que más favorece la pertenencia a una entidad deportiva es el hecho de practicar deporte. Asimismo, ser mujer también favorece la demanda analizada. Cabría esperar que fuese más probable que un hombre perteneciese a una entidad deportiva por el mayor peso específico que, en general, tiene el deporte masculino. Sin embargo, tal y como ha quedado demostrado, existen razones por las que la mujer tiene una influencia mayor, que podrían recogerse en dos causas: la gran popularidad de deportes dirigidos a este género en los gimnasios y la pertenencia a una entidad deportiva para acompañar a los hijos a actividades extraescolares.

Además, tener estudios, percibir la forma física propia como buena o residir en determinadas comunidades autónomas son otras variables que favorecen la 
pertenencia a una entidad deportiva. En esta línea, Rhodes et al. (1999) identifican una relación positiva entre el nivel de estudios y la práctica física resaltando la importancia de las influencias sociales y familiares en el ámbito deportivo. Del mismo modo, en la práctica deportiva resulta fundamental el ejemplo recibido por parte de los padres (García Ferrando, 1991) y la zona de residencia (Martínez del Castillo et al., 2006).

Todos los resultados obtenidos corroboran que la práctica deportiva es uno de los agentes de socialización de mayor interés (García Ferrando, 1991) y que las entidades deportivas son organizaciones claves en este proceso de socialización (Gómez et al., 2007). Por este motivo, resultaría interesante completar este trabajo con un estudio que analizase de manera individual las entidades deportivas públicas y privadas, con el fin de identificar las diferencias entre ambas.

Además, hay que tener en cuenta que el ámbito deportivo y en particular las organizaciones deportivas se hallan fuertemente condicionados por la situación política, social y económica, por lo que el estudio temporal de la evolución del efecto de los factores analizados en este trabajo resultaría sin duda muy enriquecedor.

Por último, con el objetivo de aunar los estudios que relacionan socialización y actividad deportiva con aquellos que asocian salud y actividad deportiva, se podría intentar vincular la encuesta sobre los "Hábitos Deportivos de los Españoles" y la Encuesta Nacional de Salud.

A la vista de los resultados obtenidos, se sugiere completar los actuales programas de promoción deportiva en edad escolar, en el deporte universitario o en el femenino, con el fomento de la actividad deportiva en los sectores sociales más desfavorecidos o en las comunidades autónomas con menor tradición deportiva.

\section{REFERENCIAS BIBLIOGRÁFICAS}

ARAGÓN, P. (1992): "Técnicas de dirección y marketing para entidades deportivas", Cuadernos técnicos Unisport, 18. Málaga.

ARRUZA, J. et al. (2008): "The impact of duration in sport and physical activity on the psychological well-being", Revista Internacional de Medicina y Ciencias de la Actividad Física y el Deporte, 8 (30): 171-183.

BALAGUER, I. y CASTILLO, I. (2002): "Actividad Física, ejercicio físico y deporte en la adolescencia temprana", en I. Balaguer (2002) (eds.), Estilos de vida en la adolescencia, (37-64), Valencia: Promolibro. 
BARROS, C.P. et al. (2007): "Sponsorship brand recall at the euro 2004 soccer tournament", Sport Marketing Quarterly, 16 (3): 161-170.

BAZO, M. T. (1996): "Aportaciones de las personas mayores a la sociedad: Análisis sociológico", Revista Española de Investigaciones Sociales, 73: 209-222.

BREUER, C. and SCHLESINGER, T. (2006): "Altern und Sportartikelnachfrage [Ageing and the demand for sporting goods]", Sport und Gesellschaft-Sport Soc, 3 (2):175-197.

BREUER, C. et al. (2010): "Socio-economic patterns of sport demand and ageing", European Review of Aging and Physical Activity 7 (2): 61-70.

BUÑUEL A. (1994): "La construcción social del cuerpo de la mujer en el deporte”, Revista Española de Investigaciones Sociológicas, 68: 97-117.

CAMPOS, A. (2005): "Situación profesional de las personas que trabajan en funciones de actividad física y deporte en la Comunidad Autónoma Valenciana (2004)", Valencia: Universidad de Valencia.

CAMPOS, A. et al. (2010): "Las personas mayores y los profesionales de la actividad física y del deporte en España", Revista MHSalud, 7 (1): 1-12.

CAMPOS, A. et al. (2011): "Demanda y percepción del monitor de las personas mayores en la actividad física y deporte en España", Revista de Psicología del Deporte, 20 (1): 61-77.

CANCELA, J. M. y PARIENTE, S. (2008): "Análisis de los hábitos nutricionales y de actividad física en relación con el estado de salud y obesidad en la adolescencia. Estudio de Caso", KRONOS, Rendimiento en el deporte, 7 (13): 4-13.

COLLINS, M. F. (2003): "Social Exclusion from Sport and Leisure", en B. Houlihan (eds.), Sport and Society, (67-88), Boston: Little Brown.

CONSEJO DE EUROPA (1995): "El trabajo del Consejo de Europa en materia de deporte 1992-1993. Volumen III", Madrid: Ministerio de Educación y Ciencia. Consejo Superior de Deportes.

DELGADO, M. A. (2002): "Las concepciones previas de las personas mayores acerca de la actividad física como base para la intervención didáctica", en A. Merino, J. De la Cruz, P. Montiel (coords.), $1^{\circ}$ Congreso Internacional de actividad física y deportiva para personas mayores (227-262), Málaga: Diputación de Málaga.

DEL HIERRO, D. et al. (2008): "El gasto en actividad física de las personas mayores en España. La demanda establecida”, Active Training, 24: 32-33.

ERBEN, R.; FRANZKOWIAK, P. y WENZEL, E. (1992): "Assessment of the outcomes of health intervention", Social Science and Medicine, 35: 359-365.

ESNAOLA, I. et al. (2011): "Relación entre variables psicosociales y la salud percibida", Revista de Psicología del Deporte, 20 (2): 413-427. 
FERNÁNDEZ-BALLESTEROS, R. (1997): "Quality of life: concept and assessment" in J. Adair, D. Belanger y K. Dion, Advances in Psychological Science (I), Social, Personal and Cultural Aspects, Montreal: Psychological Press.

FERNÁNDEZ-BALLESTEROS, R. et al. (2004): "Assessing competence: the European Survey on Aging Protocol (ESAP)", Gerontology, 50: 330-347.

FERNÁNDEZ-BALLESTEROS, R. et al. (2007): "Quality of life, Life satisfaction, and Positive Aging" in R. Fernández- Ballesteros (ed.), Geropsychology, European Perspective for an Aging World, (197-223), Gottingën: Hogrefe \& Huber.

FOX, J. and MONETTE G. (1992): "Generalized colinearity diagnostics", Journal of the American Statistical Association, 87: 178-183.

FRICK et al. (2010): "Analysing head coach dismissals in the German bundesliga with a mixed logit approach", European Journal of Operational Research, 200 (1): 151-159.

GARCÍA FERRANDO, M. (1990): Aspectos sociales del deporte. Una reflexión sociológica, Madrid: Alianza.

GARCÍA FERRANDO, M. (1991): Los españoles y el deporte (1980-1990). Un análisis sociológico, Madrid: Instituto de Ciencias de la Educación Física y el Deporte.

GARCÍA FERRANDO, M. (2001): Los españoles y el deporte: prácticas y comportamientos en la última década del siglo XX, Madrid: MECD-CSD.

GARCÍA FERRANDO, M.; LAGARDERA, F. y PUIG, N. (2002): "Cultura deportiva y socialización", en García Ferrando, N. Puig y F. Lagardera (comps.), Sociología del Deporte (69-96), Madrid: Alianza Editorial.

GARCÍA LANZUELA, Y. et al. (2007): "Sedentarismo y percepción de la salud: diferencias de género en una muestra aragonesa", Revista Internacional de Medicina y Ciencias de la Actividad Física y el Deporte, 28 (7): 344-358.

GÓMEZ, S.; OPAZO, M. y MARTÍ, C. (2007): "Características estructurales de las organizaciones deportivas", Documento de Investigación $n^{\circ} 704$, IESE Bussiness School-Universidad de Navarra.

GONZÁLEZ, M. D. et al. (2008): "Las necesidades de actividad física de las personas mayores en España. Diferencias según condición social”, Habilidad motriz, 31: 16-24

GREENE, W.H. (2011): Econometric Analysis (7 ed.), New Jersey: PrenticeHall.

JAMET, M. (1998): "Changing Patterns of Sporting Practice in France", International Review for the Sociology of Sport, 33(2): 183-187.

JIMÉNEZ-BEATTY, J. E. (2002): "La demanda de servicios de actividad física en las personas mayores". Tesis doctoral no publicada. León: Universidad e León. 
LERA-LÓPEZ, F. and RAPÚN-GÁRATE, M. (2007): "The demand for sport: sport consumption and participation models", Journal of Sport Management, 21: 103-122.

LONG, J. S. (1997): Regression Models for Categorical and Limited Dependent Variables (Advanced Quantitative Techniques in the Social Sciences), London: Sage Publications.

MARTÍNEZ DEL CASTILLO, J. et al. (2006): "Condiciones de vida, socialización y actividad física en la vejez", Revista Internacional de Sociología, 44: 39-62.

McFADDEN (1981). "Econometric Models of Probabilistic Choice", in C.F. MANSKI and D. MCFADDEN (eds.), Structural Analysis of Discrete Data with Econometric Applications, (198-272), Cambridge, MA: MIT Press.

McGUIRE, F. A. (1985): "Constraints in Later Life", en M. G. Wade (eds.), Constraints on Leisure", (335-353), Springfield: Charles C. Thomas.

MOSQUERA, M. J. y PUIG, N. (2002): "Género y edad en el deporte", en M. García Ferrando, N. Puig y F. Lagardera (comps.), Sociología del Deporte (114-141), Madrid: Alianza Editorial.

MYERS, R. H. (1990): Classical and Modern Regression with Applications, Boston: PWS-KENT Publising Company.

PATRIKSSON, G. (1996), "Síntesis de las investigaciones actuales: segunda parte" en VV.AA., La función del deporte en la sociedad: salud, socialización y economía (123-149), Madrid: Consejo de Europa-Consejo Superior de Deportes.

PAVÓN, A. y MORENO, J. A. (2008): "Actitud de los universitarios ante la práctica físicodeportiva: diferencias por géneros", Revista de Psicología del Deporte, 17 (1): 7-23.

PAWLOWSKI, T. and BREUER, C. (2011): "The demand for sports and recreational services: Empirical evidence from Germany", European Sport Management Quarterly, 11 (1): 5-34.

PONS, D. (1998): "Vivir activos para vivir mejor. Estilos de vida activos en la tercera edad", Información Psicológica, 67: 18-21.

POWELL, K. E. et al. (1987): "Physical activity and the incidence of coronary heart disease", Annual Review of Public Health, 8: 253-287.

PUIG, N. y HEINEMANN, K. (1991): "El deporte en la perspectiva del año 2000", Papers de Sociologia, 38: 123-141.

PUIG, N. y SOLER, S. (2004): "Mujer y deporte en España: Estado de la Cuestión y Propuesta Interpretativa", Apunts: Educación Física y Deportes, 76: 71-78.

RHODES, R. E. et al. (1999): "Factors associated with exercise adherence among older adults: an individual perspective", Sports medicine, 31 (4): 439454. 
RODRÍGUEZ, A. (2008): "Los recursos financieros de las entidades deportivas de la comunidad de Madrid: especial consideración de las subvenciones públicas para la promoción de actividades deportivas", Revista internacional de derecho y gestión del deporte, 3: 11-26.

SANDLER, T. and TSCHIRHART, J. (1997): "Club theory: thirty years later", Public Choice, 93 (December): 335-355.

SCHEMPP, P. G. (1997): "Pedagogical knowledge in physical education" in G. Doll-Tepper (eds.), Physical Education and Sport: Changes and Challenges, Berlin, Germany: Free University.

SERRANO, J. A. (1999): "Prácticas y audiencias deportivas. Una exploración de la consistencia y dirección de sus relaciones", Revista Española de Investigaciones Sociológicas, 87: 101-134.

SHEPHARD, R. J. (1994): "Determinants of Exercise in People Aged 65 Years and Older", en R. Dishman (eds.), Advances in Exercise Adherence, (344360), Champaign: Human Kinetics Publishers.

SLACK, T. and PARENT, M. M. (2006): Understanding sport organitations: The application of organization theory, Champaign, IL.: Human Kinetics.

SOBEJANO, I. et al. (2007): "Estudio poblacional de actividad física en tiempo libre", Gaceta Sanitaria, 23(2): 127-132.

TAKS, M.; RENSON, R. and VANREUSEL, B. (1999): "Consumer expenses in sport: a marketing tool for sports and sports facility providers?", European Journal for Sport Management, 6 (1): 4-18.

TITTEL, K. e ISRAEL, L. (1991): "La inactividad física aumenta los factores de riesgo para la salud y la capacidad física (Declaración de posición de la Federación Internacional de Medicina del Deporte - FIMS)", Boletín Femede, 12: 2-3. 